\title{
Infantile spasms syndrome in monozygotic twins
}

\author{
L PAVONE, F MOLLICA, GEMMA INCORPORA, AND G PAMPIGLIONE \\ Department of Paediatrics, University of Catania, Italy, and The Hospital for Sick Children, London
}

SUMMARY The infantile spasms syndrome appeared on the same day in a pair of monozygotic twins at age 6 months. Clinical, electroencephalographic, and neuroradiological findings during the development of the disease are reported. One of the twins was treated with ACTH and in his case clinical and electroencephalographic improvement was more rapid than in the other who was treated with clonazepam. While the computer tomography scan initially showed in each twin an area of low density in the right fronto-parietal region, this had disappeared in the tomograms obtained about 8 months later.

The infantile spasms syndrome (also called West's syndrome) ${ }^{\mathbf{1}}$ is a characteristic form of epilepsy occurring in early infancy, which presents with massive but brief flexor or extensor spasms recurring at intervals of several seconds. Mental and motor regression usually becomes rapidly obvious. In most cases the electroencephalographic (EEG) findings are those of 'hypsarrhythmia'. Follow-up studies have shown that the prognosis is generally poor with a fairly high rate of mortality in the first 10 years of life, and sequelae of mental and psychiatric disorders. ${ }^{1-2}$ The precise aetiology of the syndrome is not known but some authors have divided the group into 'cryptogenic' (if no previous illness is apparent), or 'secondary' (if pre-existing disorders affecting the brain are present). In either group however, anatomical alterations of the brain are frequently found whether by neurological investigations (pneumoencephalography) or computerised tomography (CT) scan. The syndrome is usually sporadic and familial cases have been infrequent.

The present report concerns a couple of monozygotic twins in whom, on the same day, at age 6 months, infantile spasms appeared. One of the twins was treated with ACTH and the other with clonazepam with a follow-up of 2 years.

\section{Clinical, EEG, and neuroradiological aspects}

A distant relative of the father was mentally retarded and was said to suffer from generalised convulsions, but the family history did not show any other neurological or mental disorder in close relatives. The parents were unrelated and in good health; both were schoolteachers. The first pregnancy had been interrupted by a spontaneous abortion during the second month of gestation. During the second pregnancy (at about 10 weeks' gestation), the mother suffered from a febrile illness, said to be flu, of 2 or 3 days' duration, which was treated with aspirin. There were no other problems during pregnancy and spontaneous delivery occurred at 35 weeks with 2 apparently normal male twins, each weighing about $2000 \mathrm{~g}$. There were no complications after delivery, and the neonatal period was normal with a mild physiological jaundice that started at age 3 days of very short duration. At ages 3 and 4 months both babies received oral polio vaccine.

By age of about 4 months, the parents felt that both babies' psychomotor development was perhaps not as rapid as they hoped. At age 6 months, on the same day, and with an interval of only a few hours, both infants developed typical brief massive spasms of the limbs, in flexion, and recurring at intervals of several seconds with bouts of 15 to 20 spasms. Each bout recurred several times a day until the age of about 8 months when eventually the infants, still untreated, were referred to the Department of Paediatrics at the University of Catania, Italy.

The physical development of both twins appeared normal (weight and height just below the 50th centile) with normal head circumference. Psychomotor development however, was poor. Both infants had no smile, little facial expression, and appeared lethargic with little if any response to environmental stimuli. The Moro reflex was still present and both infants showed hyper-reflexia but were otherwise floppy, unable to sit alone, or to grasp objects with either hand. In addition to the great similarity of physical and behavioural features, the clinical impression of monozygosis was supported through analysis of the dermatoglyphic patterns, haptoglobin 
groups, and of the blood groups $\mathrm{ABO}, \mathrm{Rh}, \mathrm{MNS}$, Kell, and Duffy which were identical in both infants.

Laboratory investigations gave results within normal limits for serum calcium, plasma sodium, chloride, plasma urea, blood sugar, and alkaline phosphatase. Amino-acid chromatography was normal both in the serum and in the urine, and tests for toxoplasmosis, cytomegalovirus, and rubella were negative. Ophthalmological examination was normal. The first EEG (Figure, top and bottom left) showed a gross irregularity of patterns with absence of the normal rhythmic activity and multifocal discharges. A CT scan taken a week after admission showed an area of low density in the right frontoparietal region in both infants; this was initially interpreted as evidence of some degree of cortical atrophy.

It was decided that each twin should have a different type of treatment. Twin 1 was given clonazepam $0.4 \mathrm{mg} /$ day; after 10 days the bouts of spasms were less frequent and the EEG changes less severe (Figure, top middle). However, after 40 days of treatment the spasms were still present and the EEG still showed considerable abnormalities (Figure, top right).

Twin 2 was given, instead, ACTH 25 units/day for the first 20 days and every 48 hours for the next 20 days. This was followed by maintenance therapy with oral prednisolone. At the end of the first week of this treatment the bouts of spasms became infrequent and then disappeared altogether after 2 weeks of treatment. In the EEG there were still clear alterations after 10 days (Figure, bottom middle), but a pronounced improvement was seen in the EEG taken 40 days after the beginning of treatment (Figure, bottom right) with appearance of normal rhythmic activity.

The infants are now 2 years old and are treated with sodium valproate, $4 \mathrm{mg} / \mathrm{kg}$ per day. Twin 1 still has infrequent grand mal attacks, while twin 2 is seizure-free. Psychomotor development has been limited in both twins with an IQ of about 70 on the Weschler scale. In both patients, the CT scan
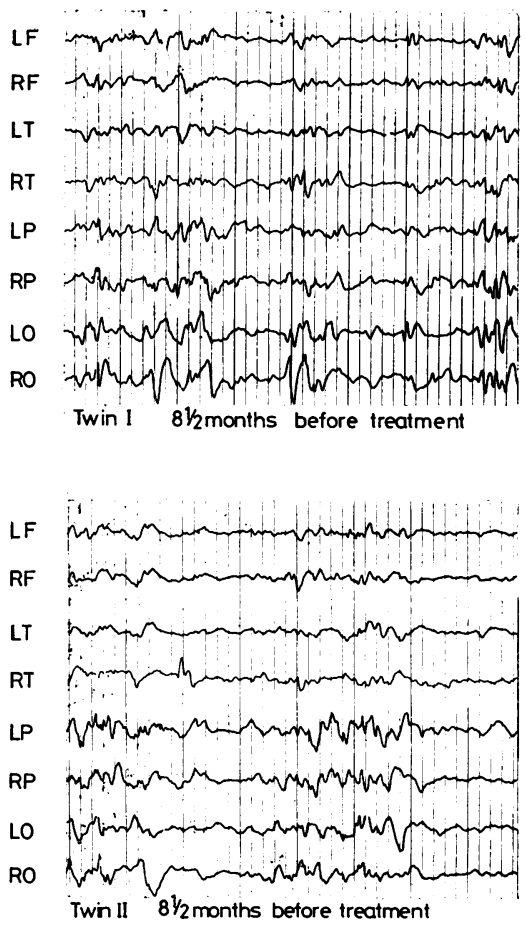


Figure EEGs of the twins recorded before, and 10 days, and 40 days, after the beginning of treatment (calibration $5 \mathrm{~mm} / 50 \mu \mathrm{V} ;$ speed $3 \mathrm{~cm} /$ second time constant $0 \cdot 1 \cdot$ second). 
repeated 8 months after the first showed a slight dilatation of the lateral ventricles and of the third ventricles with a wider inter-hemispheric area, while the low density zone in the right fronto-parietal region had completely disappeared.

\section{Discussion}

Three aspects of this pair of twins are of much interest: (1) The occurrence of infantile spasms has not been previously reported in monozygotic twins with simultaneous appearance of the attacks. (2) In each twin there was a disappearance over a period of 8 months of CT scan changes. (3) Treatment with ACTH was more effective than that with clonazepam in the control of spasms and also gave greater improvement in the EEG features.

The precise mechanisms underlying the syndrome of infantile spasms are still unknown despite the fact that a variety of genetically-determined conditions (in particular tuberous sclerosis) have been described. Familial cases of this syndrome therefore are not surprising. However, a clear genetic marker has not yet been found. ${ }^{3}$ Nevertheless the syndrome is almost invariably sporadic as shown by the largest reported series, ${ }^{2}{ }^{4-5}$ and only a few cases of affected siblings have been reported. ${ }^{2}{ }^{4-8}$ Some of these cases are 'cryptogenic' but others are said to be associated with brain malformations, metabolic disorders, or intercurrent illnesses. In this pair of monozygotic twins, the spasms began on the same day with an interval of only a few hours without any apparent external triggering factor. This would suggest some kind of 'preprogrammed' type of disease.

The CT scan images of localised low density in the right fronto-parietal region at age 6 months, and their subsequent development with disappearance of the localised alteration $\mathbf{8}$ months later, are difficult to explain either in terms of growth and development or in terms of changes of cerebral flow. Abnormal CT scan features in infantile spasms are common and no doubt we shall learn more in the next few years when technical improvements to the scanner will provide a better definition of small lesions. No serial CT studies have been reported of children with infantile spasms.

The complete disappearance of localised CT scan changes in our patients should suggest caution in the interpretation of the underlying processes, and particularly if the changes are interpreted as underlying cortical atrophy which is often considered an irreversible phenomenon. ${ }^{9}$

In the treatment of infantile spasms, ACTH is generally believed to be the most effective drug available, in spite of its side effects and the possible complications on withdrawal. In the present report, twin 2 treated with ACTH improved much more rapidly than twin 1 treated with clonazepam. Since the twins were monozygotic, living together, and exposed to the same environmental factors, our results would support the idea that ACTH is more beneficial than clonazepam treatment, at least initially. Several colleagues have used a combination of ACTH and nitrazepam with some benefit.

\section{References}

1 West W J. Letter: On a peculiar form of infantile convulsions. Lancet 1840-1; i: 724-5.

2 Jeavons $\mathbf{P} \mathbf{M}$, Bower B D. Infantile spasms. Clinics in Developmental Medicine No 15. London: Heinemann, 1964: 82.

3 Howitz P, Platz P. Infantile spasms and HLA antigens. Arch Dis Child 1978; 53: 680-2.

- Thieffry St, Aicardi J. Les spasmes en flexion du nourrisson: 36 observations; étude clinique. Ann Pediatr 1958; 19: 187-98.

5 Fleiszar K A, Daniel W L, Imrey P B. Genetic study of infantile spasms with hypsarrhythmia. Epilepsia 1977; 18: 55-62.

6 Cotte-Rittaud M R, Delafin J. Présentation d'une observation familiale de maladie des spasmes en flexion avec hypsarrhythmia. Rev Neurol (Paris) 1965; 112: 301-3.

7 Bignami A, Maccagnani F, Zappella M, Tingey A $\mathrm{H}$. Familial infantile spasms and hypsarrhythmia associated with leucodystrophy.J Neurol Neurosurg Psychiatry 1966; 29: 129-34.

8 Cao A, Cianchetti C, Signorini E, Loi M, Sanna G, De Virgiliis S. Agenesis of the corpus callosum, infantile spasms, spastic quadriplegia, microcephaly, and severe mental retardation in three siblings. Clin Genet 1977; 12: 290-6.

9 Lagenstein I, Willig R P, Kuhne D. Letter: Reversible cerebral atrophy caused by corticotrophin. Lancet 1979; i: $1246-7$.

Correspondence to Dr G Pampiglione, Department of Neurophysiology, The Hospital for Sick Children, Great Ormond Street, London WC1N 3JH.

Received 16 October 1979 